\title{
A note on style
}

The endnotes in this book follow the system of citation contained in THE BluebooK: A Uniform System of Citation (19th ed. 2010). These endnotes can be considered a list of references for each of the chapters. In some instances where a single source is used to support a paragraph or series of paragraphs, citations may be limited. 